\title{
Repertoire of morphable proteins in an organism
}

\author{
Keisuke Izumi ${ }^{1}$, Eitaro Saho $^{1}$, Ayuka Kutomi ${ }^{1}$, Fumiaki Tomoike $^{1}$, Tetsuji Okada ${ }^{\text {Corresp. } 1}$ \\ ${ }^{1}$ Department of Life Science, Gakushuin University, Tokyo, Japan \\ Corresponding Author: Tetsuji Okada \\ Email address: tetsuji.okada@gakushuin.ac.jp
}

All living organisms have evolved to contain a set of proteins with variable physical and chemical properties. Efforts in the field of structural biology have contributed to uncovering the shape and the variability of each component. However, quantification of the variability has been performed mostly by multiple pair-wise comparisons. A set of experimental coordinates for a given protein can be used to define the "morphness/unmorphness". To understand the evolved repertoire in an organism, here we show the results of global analysis of more than a thousand $E$. coli proteins, by the recently introduced method, distance scoring analysis (DSA). By collecting a new index "UnMorphness Factor" (UMF), proposed in this study and determined from DSA for each of the proteins, the lowest and the highest boundaries of the experimentally observable structural variation are comprehensibly defined. The distribution plot of UMFs obtained for $E$. coli represents the first view of a substantial fraction of non-redundant proteome set of an organism, demonstrating how rigid and flexible components are balanced. The present analysis extends to evaluate the growing data from single particle cryo-electron microscopy, providing valuable information on effective interpretation to structural changes of proteins and the supramolecular complexes. 
2 Repertoire of morphable proteins in an organism

3

4 Keisuke Izumi, Eitaro Saho, Ayuka Kutomi, Fumiaki Tomoike, Tetsuji Okada

Department of Life Science, Gakushuin University, 1-5-1 Mejiro, Toshima-ku, Tokyo 171-8588, Japan.

Corresponding author: Tetsuji Okada, Department of Life Science, Gakushuin University, 1-5-1 Mejiro, Toshima-ku, Tokyo 171-8588, Japan.+81-3-3986-0221, tetsuji.okada@gakushuin.ac.jp

\section{ABSTRACT}

All living organisms have evolved to contain a set of proteins with variable physical and chemical properties. Efforts in the field of structural biology have contributed to uncovering the shape and the variability of each component. However, quantification of the variability has been performed mostly by multiple pair-wise comparisons. A set of experimental coordinates for a given protein can be used to define the "morphness/unmorphness". To understand the evolved repertoire in an organism, here we show the results of global analysis of more than a thousand $E$. coli proteins, by the recently introduced method, distance scoring analysis (DSA). By collecting a new index "UnMorphness Factor" (UMF), proposed in this study and determined from DSA for each of the proteins, the lowest and the highest boundaries of the experimentally observable structural variation are comprehensibly defined. The distribution plot of UMFs obtained for $E$. coli represents the first view of a substantial fraction of non-redundant proteome set of an organism, demonstrating how rigid and flexible components are balanced. The present analysis extends to evaluate the growing data from single particle cryo-electron microscopy, providing valuable information on effective interpretation to structural changes of proteins and the supramolecular complexes. 


\section{INTRODUCTION}

Self-replicating (living) species are defined by the presence of a genome that is used to produce a set of proteins and RNAs. The diversity of living entities, from unicellular bacteria to mammals, primarily corresponds to the varying repertoire of proteins. A proteome set limits the morphology and functionality of an organism. Therefore, understanding the atomic details of the folded state and the variability of the fold for each protein in an organism is a long-term goal in structural biology.

We have previously shown that comparison of different proteins and protein families with respect to their structural variability can be comprehensively performed by distance scoring analysis (DSA), in which all intramolecular $\mathrm{C}_{\alpha}-\mathrm{C}_{\alpha}$ pair distance variations (score $=$ average $/ \mathrm{stdev}$ ) are considered (Anzai et al., 2018) (Fig. 1). DSA is advantageous in terms of reproducibility because no procedures/assumptions associated with superimposition are involved. Also, the amount of numeral usage is far more than the pair-wise based analysis. For example, if we calculate an average root-mean-square deviation (RMSD) of $\mathrm{C}_{\alpha}$ positions for 10 structures (chains) of 100 amino acids, the number of used deviation values is 100 positions $\times 45$ structure pairs $=4,500$; whereas, DSA uses $(100 \times 99 / 2) \mathrm{C}_{\alpha}-\mathrm{C}_{\alpha}$ pair distances $\times 10$ structures $=49,500$ distance values. Furthermore, inclusion of new data is simpler in DSA as no pairing is required. Average score for a protein of 100 amino acids is thus obtained as an average of 4,950 scores from 49,500 distances in the case of 10 chain usage. Here we introduce "UnMorphness Factor" (UMF), which is defined as a converged average score for a protein, is an alternative to conventional RMSD-based comparison. In most cases, the average score of a protein decreases as the number of used chains increases, reaching a nearly steady level, which is assigned as UMF of a protein. Thus, when we successively calculate the average scores using $3,4,5 \ldots 9$ chains as average score $3,4,5, \ldots 9$, the last average score for 9 chains (average score 9) is defined as UMF (Fig. S1). In the following study, UMFs calculated from X-ray crystallography are denoted as XUMF and those from singleparticle cryo-electron microscopy (cryo-EM) are denoted as eUMF.

E.coli proteins are the most abundant prokaryotic content in the Protein Data Bank (PDB) (Burley et al., 2017). We suppose that species-based construction of a "morphability" map is most achievable, covering a substantial portion of proteins that construct the E. coli proteome. The present work is intended to assign a UMF to a protein having a UniProt ID as well as to highlight how experimental coordinates can be used to uncover the repertoire of morphable proteins in $E$. coli. Here we demonstrate that a substantial fraction $(\sim 23 \%)$ of the E.coli proteome can be 
62 described as exhibiting "morphness/unmorphness" distribution with well-defined lower/upper

63 limits, and a major cluster of data points. The result is compared to a similar amount $(\sim 1000)$ of

64 randomly selected human proteins, a part of which was the subject of our previous study (Anzai

65 et al., 2018), providing unprecedented insight into the morphness repertoire for a set of natural

66 polypeptides. These analyses of X-ray structures are also used to evaluate the recently

67 accumulating cryo-EM structures.

68

69

\section{METHODS}

\section{Protein lists}

ECOLI and HUMAN reference proteome set obtained from UniProt (The UniProt Consortium, 2017) were initially used to search for the corresponding PDB entries to each of the UniProt IDs. For ECOLI, all UniProt IDs were checked to see how many PDB entries could be found for each protein, and then the protein list was sorted by amount of entries, showing that about $30 \%$ of the ECOLI proteins have at least one X-ray entry, and roughly a half of those have more than three entries. Drill down search by organism in the PDB was also used to supplement the protein list of E. coli outside the ECOLI proteome set. In fact, about $30 \%$ of E. coli PDB entries are of the UniProt IDs not found in the ECOLI set. These entries are used if they are of the structures of virtually identical sequences to any of the ECOLI set proteins. Thus, some of 980 xUMFs of ECOLI set are calculated from multiple UniProt IDs of almost identical sequences. For HUMAN, drill down search list was primarily used after UniProt ID sorting to find out which proteins are rich in the PDB entries. Roughly 23\% ( 4700) of HUMAN reference set and reviewed proteins were found to have at least one X-ray PDB entry. This means that the number of targets amenable for DSA is fairly more than the presently performed $\sim 1000$ if we carry out the analysis as extensively as has been done for ECOLI set.

\section{Archiving}

90 Coordinate directories and data directories for proteins are separately made per UniProt ID under 91 the corresponding organism top directory. Each coordinate directory contains the raw pdb files 92 assigned to the respective UniProt ID. The data directory of each protein holds the following basic 93 file set: (1) list file, containing information of PDB entries (chain IDs, space group, lattice 
94 constants, resolution, etc), the polypeptide range (initial and final sequence positions) selected for

95 DSA, and the order of entry/chain usage in the progress plot (Anzai et al., 2018). (2) Distance file,

96 containing all $\mathrm{C}_{\alpha}$ pair position numbers and the corresponding distances. (3) Score file, containing

97 all $\mathrm{C}_{\alpha}$ pair position numbers and the corresponding average distances, stdevs, scores, maximum

98 and minimum distances. (4) Main plot scatter chart (and/or semi-log main plot scatter chart). (5)

99 Progress plot table, and (6) progress plot scatter chart.

100 To date, over 200 species or group of species ( $\sim 80$ eukaryotic, $\sim 110$ bacterial, $\sim 20$ archaeal, and

$101 \sim 15$ viral) have been archived and the total number of proteins analyzed so far exceeds 5500,

102 although the degree of DSA completeness varies among the species. Completeness means how

103 many multiple chains were included per entry. For example, our previous analysis of 300 human

104 proteins used only one chain per entry because we selected the proteins having at least four entries.

105 Although multiple chain usage from an entry do not always affect the UMFs substantially, there

106 are some cases where many chain As from distinct entries are very similar to each other but chains

107 A and B differs from each other. Thus, some of the xUMFs of human proteins would exhibit lower

108 values when our chain usage completeness becomes higher.

109 The tables of protein lists associated with the present study are provided as Supplemental files

110 (Table S1 and S2). The raw data files for E. coli and human proteins are stored in Mendeley Data.

111 An example of the list files (stored as ***_list_****.xlsx in each protein's raw data folder)

112 described above is provided as Table S3 in which the data of the cell O1, DSA123(110-232),

113 indicates that we have analyzed continuous 123 amino acid chains of the sequence number from

114110 to 232 . The numbers in the $\mathrm{O}$ column show the order of data inclusion and correspond to the

$115 \mathrm{x}$-axis of the progress plot (stored as ave-score_***.png).

116

\section{Data processing}

118 DSA procedure was performed the same as previously described (Anzai et al., 2018). A Python

119 script score-analyzer v22 has been modified from the previous version, but the way of calculation

120 of $\mathrm{C}_{\alpha}$ distances (Euclidian) remains unchanged, being compatible with commonly used protein

121 crystallography tools. Among many minor changes, such as the output function of a semi-log main

122 plot chart, for example, up to 1,000,000 $\mathrm{C}_{\alpha}-\mathrm{C}_{\alpha}$ pairs (over 1400 amino acid length) can be

123 processed now, covering almost all of the currently deposited and continuously modeled

124 structures. The longest polypeptide analyzed so far is the human U5 small nuclear

125 ribonucleoprotein $200 \mathrm{kDa}$ helicase (1397 residues, $65.4 \%$ of the full length is continuously 
126 modeled). In the previous study, the longest polypeptide analyzed was 919 amino acids (90.2\% of

127 the full length) of insulin-degrading enzyme.

128 The 7TM structures for CNR1, a G protein-coupled receptor, were obtained from our archive at 129 gses.jp/7tmsp. Each of the chains contains 200 amino acids, defined previously (Okada, 2012) for

130 reproducible comparison of all receptors including non-rhodopsin-like GPCRs.

131

\section{Mapping of UMFs to the proteome set}

133 The protein list of ECOLI and HUMAN reference (reviewed) sets obtained from UniProt were 134 used as a template. Sequence lists (FASTA) of these reference sets were used for the online 135 analysis by SCAMPI (Peters et al., 2016) to assign probable membrane proteins and the result was 136 merged to the protein template list. The coverage of the present UMFs in the ECOLI set was 137 evaluated first by trying to map all 1178 E. coli xUMFs to any of the proteins on the template list, 138 resulting in 980 non-redundant assignments. For cryo-EM data, 57 out of 58 eUMF were assigned 139 to the proteins on the template list, including 31 redundant assignment with the xUMF. In many 140 cases of these 31 proteins, eUMF was found to be substantially lower than the corresponding $141 \mathrm{xUMF}$, regardless of the number of chains used for analysis. When we mapped all $1043 \mathrm{xUMFs}$ 142 in the HUMAN reviewed reference set, no redundancy was found.

\section{RESULTS}

145

\section{UMF of proteome set}

147 Since many proteins are deposited to the PDB from a variety of $E$. coli strains, we chose a reference 148 proteome set called ECOLI (strain K-12) in UniProt. According to UniProt reference proteome sets, the proteome size ranges from 4232 (strain 333) to 6593 proteins (strain NCTC13148). Thus, the number of proteins in the ECOLI reference set (4391) is likely to be close to the minimum number necessary for constituting this prokaryotic organism.

152 As of July in 2019, the PDB contains over 8500 X-ray entries that include E.coli components

153 (mostly proteins). We calculated xUMFs for all E. coli strains, totaling 1178 proteins, including 154 redundancy in the case of some proteins. An example of this protein redundancy is $\beta$-lactamase, 155 for which many UniProt IDs are found in the various strains of E. coli. Thus, 1178 xUMF values 156 were mapped to the reference proteome set ECOLI, resulting in 980 proteins or $22.3 \%(980 / 4391)$ 157 coverage of the whole reference proteome. On the other hand, when we re-calculated the coverage 
158 separately for soluble and membrane proteins assigned by SCAMPI, a significant difference was 159 found; 26.9\% (887 / 3300) for soluble proteins and 8.5\% (93 / 1091) for membrane proteins. Thus, 160 our present analysis provides xUMFs over a quarter of soluble proteins in E. coli K-12. The 161 average ratio of the analyzed to the full length (including signal/propeptide sequences) for 980 162 proteins is $83.6 \%$ (only $6.7 \%$ of 980 proteins is limited to $<50 \%$ of full length).

163 For comparison, we have updated the previous human protein analysis and collected $1043 \mathrm{xUMFs}$.

164 The SCAMPI analysis for the reviewed human reference set of 20,416 proteins assigned 6293 as 165 membrane proteins. Since 222 out of 1043 xUMFs presumably are part of the 6293 transmembrane 166 proteins, the coverage is 3.6\%; whereas, 821 out of $14123(=20416-6293)$ corresponds to $5.8 \%$ 167 coverage for human soluble proteins.

168

\section{Summary plot for $E$. coli and human proteins}

170 When all 980 xUMFs of the ECOLI set proteins are plotted against the analyzed chain length in 171 log-log scale, a fairly dispersed image can be obtained (Fig. 2A). This is a log version of the 172 previously described "summary plot" (Anzai et al., 2018). A corresponding figure for 1039 human 173 proteins is also shown in Fig. 2C. These two images are similar despite all proteins being chosen 174 randomly and only by giving selection priority based on the availability of the experimental 175 coordinates by X-ray crystallography. An obvious difference between the ECOLI set and the 176 human data is the amount of high xUMF proteins in the former and this presumably occurs because 177 xUMFs of a substantial part of the ECOLI set proteins are calculated using less than three PDB 178 entries; this means that more than one chain per entry had to be used. In fact, of the 38 proteins exhibiting xUMFs larger than 500, 34 proteins are analyzed using only one or two entries.

180 When the horizontal axis is changed to the average resolution, a cluster of proteins becomes 181 clearer. In the ECOLI plot (Fig. 2B), ribosomal proteins, which includes thirty-two 50S and twenty 182 30S subunit proteins analyzed using mostly the same set of PDB entries, are shown as a thin cluster 183 at the average resolution from 3.2 to $3.3 \AA$ and the xUMF range from 25 to 70 . On the other hand, 184 COP9 signalosome complex subunits dominate the data of average resolution lower than $4.3 \AA$ in 185 the human summary plot (Fig. 2D).

\section{Distribution plot}

188 The summary plots of ECOLI and human proteins confirmed that the lower limit of xUMFs is $189 \sim 15$, as previously reported for human calmodulin. As long as we analyze X-ray structures, xUMFs 
190 in the range from 0 to 10 (over $10 \%$ average deviations for all intramolecular $\mathrm{C}_{\alpha}-\mathrm{C}_{\alpha}$ pair distances)

191 are not likely to emerge. In fact, based on the over 5500 proteins from various species analyzed so

192 far, the lower limit of $\sim 15$ is still valid (unpublished result). Many proteins that are reluctant to

193 crystallize would contain very flexible parts. If such flexible parts dominate the entire length of a

194 polypeptide in a given protein, as can be seen in many NMR depositions (ensembles), the UMF

195 should approach to 0. Thus, many intrinsically disordered proteins (Dunker et al., 2001; Ahrens,

196 Nunez-Castilla \& Siltberg-Liberles, 2017) can be understood to exhibit 0 10 UMFs that cannot

197 be evaluated by X-ray crystallography.

198 To demonstrate the probable upper limit of xUMFs, distribution of xUMF values shown in the 199 summary plot are counted from 0 to 900 with the slot of 30 (Fig. 3). Whereas some proteins (8 out 200 of 980 E. coli proteins, and 1 out of 1039 human proteins) exhibited the xUMFs larger than 900 201 and not shown in the figure, the plot demonstrates that most proteins would rarely exceed the 202 xUMF over 500. This result means that the averaged uncertainty (standard deviation) of all $\mathrm{C}_{\alpha}-\mathrm{C}_{\alpha}$ 203 distances in a folded polypeptide chain determined by X-ray crystallography inevitably exceeds $2040.2 \%(0.1 \AA$ per $50 \AA)$.

205

206 Cryo-EM structure analysis

207 Cryo-EM data deposition is remarkably increasing in the PDB (Bai, McMullan \& Scheres, 2015; 208 Nogales, 2016; Raunser, 2017). While the resolution of data by this method rarely reaches the 209 precision that most X-ray structures can resolve, we have examined cryo-EM structures of ECOLI 210 reference proteome set and calculated eUMFs for 56 proteins of which 31 have respective xUMFs. 211 As a result, 1005 UMFs (980 xUMFs plus 25 eUMFs) out of 4391 ECORI set are obtained, 212 corresponding to $22.9 \%$ coverage. Membrane proteins make up 11 of the 23 eUMFs, reflecting 213 that cryo-EM is frequently applied to targets that are reluctant to crystallize.

214 When we add the eUMF data to the ECOLI reference set summary plot of xUMFs shown in Fig. 215 2, a clear distinction becomes obvious (Fig. 4). Importantly, eUMF can be seen more frequently 216 in the long polypeptides and in the low value ranges.

217 DSA analysis of cryo-EM structures also provided some examples that are distinct from that 218 obtained from a larger set of X-ray entries. The semi-log main plot, which is another form of the 219 previously reported main plot (Anzai et al., 2018) and is a 2D representation of highly variable 220 parts in a protein, is especially favorable in demonstrating how cryo-EM and X-ray structures can 221 explore the morphness of proteins. In Fig. 5A, a semi-log main plot is shown for the cryo-EM 
222

223

224

225

226

227

228

229

230

231

232

233

234

235

236

237

238

239

240

241

242

243

244

245

246

247

248

249

250

251

252

253

entry (PDB ID: 5Y4O) of low conductance mechanosensitive channel YnaI (Yu et al., 2018). This entry contains seven chains of identical length ( 223 residues, $65 \%$ of the full length). The unusually high eUMF of 79821.6 on this plot is explained by a large part of the polypeptide being virtually identical among the seven chains and clearly separated from the lower score thin cluster representing the $\mathrm{C}_{\alpha}$ pair distances in the transmembrane (TM) part. Obviously, the eUMF in this case is far from the expected value for this protein. Seven other proteins are also outstanding in the unusual value of eUMFs (Fig. 4, Table S1).

YnaI is functionally related to the MscS mechanosensitive channel (Böttcher et al., 2015). Although the sequence similarity between them is limited, they both self-assemble to form a heptamer, in which each protomer assumes an elongated conformation. Single entry analysis of MscS as has been done for YnaI, but using X-ray entry (PDB ID: 2OAU) (Steinbacher et al., 2007), resulted in xUMF of 190.6, which is considerably lower than eUMF of YnaI and fairly in the range shown in the distribution plot (Fig. 3). The corresponding semi-log main plot demonstrated multidomain morphness (Fig. 5C), but not separated from each other in the average score range. Multi entry analysis ( 1 chain usage per entry and 6 entry usage) lowered the xUMF to 119.0, resulting in a diffused pattern on the semi-log plot (Fig. 5B). For comparison, the pattern of semilog plot obtained for 200 residue TM bundle of a $\mathrm{G}$ protein-coupled Cannabinoid receptor 1 (CNR1) is shown in Fig. 5D. As the analyzed part is only limited to compactly arranged seven TM helices, the maximum dimension is about a half of YnaI and MscS. This receptor, whose conformational change occurs mostly in one of the seven helices (Hua et al., 2017), is among many examples where any large domain morphness does not occur but the polypeptide contains very variable parts, resulting in a low xUMF (58.9 for CNR1).

For other two proteins, ribosome hibernation promoting factor (HPF) and ribosome modulation factor (RMF) (Beckert et al., 2018), out of the eight proteins with unusually high eUMFs (Fig. 4), there were respective X-ray entries (but not enough chains for xUMF calculation). By merging just one X-ray entry with the corresponding cryo-EM entries for each of the two proteins, the resulting UMF (xeUMF) dramatically decreased to 90.4 for HPF and 66.0 for RMF, falling into range on distribution plot of ECOLI (Fig. 3, red). This result is promising to further utilization of increasing cryo-EM entries in combination with the respective X-ray entries for obtaining more UMF data for the proteins that were not amenable to DSA.

In the normal scale main plot of YnaI (Fig. 6A), $\mathrm{C}_{\alpha}$ pairs in the morphable parts are difficult to assess when we include all data points. On the other hand, $\mathrm{C}_{\alpha}$ pairs in the unmorphable parts exhibit

Peer) reviewing PDF | (2019:10:42306:2:0:NEW 25 Dec 2019) 
254 almost linear distance dependency. This type of main plot did not typically arise from a set of X-

255 ray structures, but could be seen for a polypeptide consisting of a larger multi-chain complex, such 256 as human hist1h2ab (histone H2A.2) as demonstrated previously (Anzai et al., 2018). The updated 257 main plot, adding eight entries (chains) to the previous data, of hist1h2ab is shown in Fig. 6C.

258 For prepilin peptidase-dependent pilin (PPDD) (Bardiaux et al., 2019), which is another one of 259 eight unusual eUMF value, the main plot (Fig. 6B) was found to be just like the pattern of 260 hist1h2ab. These patterns are almost artificially reproducible by using values of model standard 261 deviations for the average distances, and represent the examples of uniform $\mathrm{C}_{\alpha}$ coordinate 262 deviations at all positions in the polypeptide.

263 In Fig. 6D, the main plot of hist1h2ab by cryo-EM entries is shown. Although the used number of 264 chains (22 chains, one chain per entries) are less than a half of X-ray entries (56 chains, one chain 265 per entries), the cluster of $\mathrm{C}_{\alpha}$ pairs by cryo-EM was found mostly in the lower average scores than 266 that by X-ray data. Correspondingly, eUMF of 91.0 is lower than the xUMF of 143.5 which is 267 substantially unchanged from the previous value with 48 chains usage.

\section{Application to recent entries}

270 How newly added PDB entries exhibit different backbone structures from that of previously 271 deposited ones for the same protein is readily evaluated by the present method. Here we 272 demonstrate an example obtained recently in July of 2019.

273 SOS response-associated protein YedK (Thompson et al., 2019) is included in the ECOLI 274 reference proteome set and has a calculated xUMF of 321.7 (shown as a red circle in Fig. 7A), 275 using only three chains from two entries out of three entries deposited for UniProt ID of P76318.

276 The unused entry contains two regions of chain-break within the almost full-length model.

277 More recently, five entries were added to the PDB for the same protein but with different UniProt 278 ID of A0A2S5ZH06 (100\% identical to P76318). As the modeled length was the same as that of 279 two entries for P76318, the new distance data can be added to the previous distance data, making 280 the progress plot meaningful (shown as gray circles in Fig. 7A). The new data contributed to the 281 data point 4 to 9 , using 4 out of 5 entries and 6 chains from 4 entries (three chains from one entry 282 6KBZ). The average score value at the data point 9 translates to $x U M F$ of 161.4, which is about a 283 half of the previous 321.7. We have been making clear separation of entry usage and chain usage 284 for most of over 5500 protein analysis, and the progress plot in this case is rearranged to 6 entry 285 usage first followed by the redundant chain usage as shown in Fig. 7C where a redundantly used 
286

\section{7}

288

289

290

291

292

293

294

295

296

297

298

299

300

301

302

303

304

305

306

307

308

309

310

311

312

313

314

315

316

317

chain for P76318 is moved from data point 3 to 7 . Although such a data switch in the progress plot did not cause any significant change in this case, data order switch would be useful for other proteins to see the impact (discontinuous drop of the average score) of some specific entries/chains in a set of many entries.

Two semi-log plots, one for P76318 and the other for P76318/A0A2S5ZH06, demonstrate the effect of entry/chain addition (Fig. 7B, D). Most of the $\mathrm{C}_{\alpha}-\mathrm{C}_{\alpha}$ pairs that appeared in the average score of over 1000, moved to much lower score region, whereas the highly variable pairs with low scores of less than 10 remain largely unchanged. This indicated that the first two entries (three chains) for YedK differed significantly from each other in the high morphness regions.

\section{DISCUSSION}

In this study, proteins are selected for DSA when at least 3 chains (for E. coli) or 3 entries (for human) are available, with an exception for the case that the continuously modeled part is too short against the full length. Overall, the average usage per protein is 7.9 entries (10.1 chains) for the ECOLI reference set and 13.9 entries (15.4 chains) for the human proteins. The higher number in the human set is due to the data abundance in the PDB and to several heavily deposited proteins (such as carbonic anhydrase 2, for which we have used over 600 entries to calculate the xUMF). When we recalculate the numbers for the human set after removing the 12 proteins for which more than 100 entries/chains are used, the average usage drops to 10.9 entries (12.5 chains). For the ECOLI set, only maltose-binding periplasmic protein exceeds 100 entry/chain usage. These considerations indicate that the overall amount of data usage is not very different between the ECOLI set and the human protein set. On the other hand, a substantial portion of the ECOLI set xUMFs originates from the analysis of using only one or two entries per protein, taking multiple chains per entry. This limitation is presumably reflected in the xUMF distribution plot (Fig. 3) as discussed below. Our analysis also suggests that multiple chain usage frequently works for decreasing the UMF of a protein for which only one type of crystal lattice is available for multiple entries.

As for the summary plots (Fig. 2), xUMFs appear to exhibit only weak dependence either on the polypeptide length or on the X-ray data resolution (correlation coefficients of $0.19,-0.12,0.30$, 0.21 for panel $\mathrm{A}, \mathrm{B}, \mathrm{C}, \mathrm{D}$, respectively). We have previously reported correlation coefficients corresponding to Fig. 2D of the present study but for $\sim 100$ proteins $\times 3$ sets separated according to the amount of entry usage. The previous value of -0.33 (an average of 3 sets) decreased 
318 substantially to -0.21 for the 1039 proteins analyzed here. As a reference, similar analysis of over

3195500 proteins from various species (including E.coli and human) resulted in correlation 320 coefficients of 0.28 and -0.22 vs chain length and average resolution, respectively (unpublished 321 results). These studies are consistent with a conclusion that xUMFs are slightly more dependent 322 on the chain length than on the data resolution.

323 A clear difference between the ECOLI set and human proteins in the distribution plot (Fig. 3) is 324 the smoothness of the pattern. In addition, the peak fraction is clearer in the human plot (gray), 325 where over $20 \%$ of the 1039 proteins are in the xUMF range of 90 to 120 , corresponding to $1.1 \%$ 326 to $0.83 \%$ average uncertainty of all $\mathrm{C}_{\alpha}-\mathrm{C}_{\alpha}$ distances. The higher fraction in the UMF range roughly 327 from 250 to 400 in E. coli (red) could be due to the proteins analyzed using more than 3 chains per 328 entry. It should also be noted that the distribution plot of human was so irregular in the previous 329 analysis of a limited number of proteins that it could not be considered (Anzai et al., 2018).

330 If we roughly classify the $E$. coli proteins as flexible, average, and rigid, based on the xUMF ranges $3310-100,101-200$, and 201-, the ratio in each class is $22.9 \%, 43.0 \%$, and $34.1 \%$, respectively $(29.1 \%$, $33249.7 \%$, and $21.2 \%$ for human proteins). Differences in the xUMF values among the proteins 333 analyzed by DSA reflect the presence/absence of distinct conformational states and/or the degree 334 of backbone fluctuation in each protein.

335 Our results indicate that cryo-EM is effective in detecting conformational variability of large 336 molecular weight proteins (Fig. 4). While the clear differences in the main plot pattern between 337 cryo-EM and X-ray (Fig. 5, 6) could arise from various factors, our results suggest that cryo-EM 338 is potentially effective in uncovering the full morphness range of a polypeptide with fewer 339 structure determinations (PDB entries).

340

\section{CONCLUSIONS}

342 The growth in the structural data accumulation has been outstanding for human proteins and the 343 trend will undoubtedly continue in the future. Uncovering the morphness repertoire for any 344 eukaryotic organism is very challenging due to the presence of splice variants and intrinsically 345 disordered proteins. The total number of PDB entries for $E$. coli proteins is the second largest (after 346 human proteins) and is exceptionally large among prokaryotic organisms. The present study shows 347 that about a quarter of $E$. coli's proteome set could have already been the subject of detailed 348 morphness analysis. More coverage would be possible if we further include very similar 349 orthologues from other bacteria such as salmonella species. UMF calculation by cryo-EM data 
350 only (eUMF) was found to be unreliable in some cases but the data would undoubtedly be useful 351 especially in combination with the corresponding X-ray data. It should be noted that we have not 352 applied any resolution cut criteria for cryo-EM data analysis. While NMR data usage has not been 353 given priority in our studies so far, it will have to be done in the future.

354 All DSA results can improve on a weekly basis in accordance with updates of the PDB contents. 355 Importantly, the results presented here, when the sharing environment is established, can conform 356 to FAIR data principles (Wilkinson et al., 2016) since no assumptions are involved in the analysis. 357 Anyone can contribute to improve the quality of the data set by, for example, rationally changing 358 the selection of the entries/chains based on their expertize of specific proteins. 
361

362

363

364

365

366

367

368

369

370

371

372

373

374

375

376

377

378

379

380

381

382

383

384

385

386

387

388

389

390

391

392

References

Ahrens JB, Nunez-Castilla J, Siltberg-Liberles J. 2017. Evolution of intrinsic disorder in eukaryotic proteins. Cellular and Molecular Life Sciences 74:3163-3174.

Anzai R, Asami Y, Inoue W, Ueno H, Yamada K, Okada T. 2018. Evaluation of variability in high-resolution protein structures by global distance scoring. Heliyon 4:e00510.

Bai X, McMullan G, Scheres SHW. 2015. How cryo-EM is revolutionizing structural biology. Trends in Biochemical Sciences 40:49-57.

Bardiaux B, de Amorim GC, Luna Rico A, Zheng W, Guilvout I, Jollivet C, Nilges M, Egelman EH, Izadi-Pruneyre N, Francetic O. 2019. Structure and Assembly of the Enterohemorrhagic Escherichia coli Type 4 Pilus. Structure 27:1082-1093.e5.

Beckert B, Turk M, Czech A, Berninghausen O, Beckmann R, Ignatova Z, Plitzko JM, Wilson DN. 2018. Structure of a hibernating 100S ribosome reveals an inactive conformation of the ribosomal protein S1. Nature Microbiology 3:1115-1121.

Böttcher B, Prazak V, Rasmussen A, Black SS, Rasmussen T. 2015. The structure of ynai implies structural and mechanistic conservation in the mscs family of mechanosensitive channels. Structure 23:1705-1714.

Burley SK, Berman HM, Kleywegt GJ, Markley JL, Nakamura H, Velankar S. 2017. Protein data bank (PDB): the single global macromolecular structure archive. Methods in Molecular Biology 1607:627-641.

Dunker AK, Lawson JD, Brown CJ, Williams RM, Romero P, Oh JS, Oldfield CJ, Campen AM, Ratliff CM, Hipps KW, Ausio J, Nissen MS, Reeves R, Kang C, Kissinger CR, Bailey RW, Griswold MD, Chiu W, Garner EC, Obradovic Z. 2001. Intrinsically disordered protein. Journal of molecular graphics \& modelling 19:26-59.

Hua T, Vemuri K, Nikas SP, Laprairie RB, Wu Y, Qu L, Pu M, Korde A, Jiang S, Ho J-H, Han GW, Ding K, Li X, Liu H, Hanson MA, Zhao S, Bohn LM, Makriyannis A, Stevens RC, Liu ZJ. 2017. Crystal structures of agonist-bound human cannabinoid receptor CB1. Nature 547:468-471.

Nogales E. 2016. The development of cryo-EM into a mainstream' ' structural biology technique. Nature Methods 13:24-27.

Okada T. 2012. Comparative analysis of the heptahelical transmembrane bundles of G proteincoupled receptors. Plos One 7:e35802. 
393 Peters C, Tsirigos KD, Shu N, Elofsson A. 2016. Improved topology prediction using the terminal

394

395

396

397

398

399

400

401

402

403

404

405

406

407

408

409

410

411

412

413

414

415

416

417 hydrophobic helices rule. Bioinformatics 32:1158-1162.

Raunser S. 2017. Cryo-EM Revolutionizes the Structure Determination of Biomolecules. Angewandte Chemie 56:16450-16452.

Steinbacher S, Bass R, Strop P, Rees DC. 2007. Structures of the prokaryotic mechanosensitive channels mscl and mscs. In: Mechanosensitive ion channels, part A. Current topics in membranes. Elsevier, 1-24.

The UniProt Consortium. 2017. UniProt: the universal protein knowledgebase. Nucleic Acids Research 45:D158-D169.

Thompson PS, Amidon KM, Mohni KN, Cortez D, Eichman BF. 2019. Protection of abasic sites during DNA replication by a stable thiazolidine protein-DNA cross-link. Nature Structural \& Molecular Biology 26:613-618.

Wilkinson MD, Dumontier M, Aalbersberg IJJ, Appleton G, Axton M, Baak A, Blomberg N, Boiten J-W, da Silva Santos LB, Bourne PE, Bouwman J, Brookes AJ, Clark T, Crosas M, Dillo I, Dumon O, Edmunds S, Evelo CT, Finkers R, Gonzalez-Beltran A, Gray AJ, Groth P, Goble C, Grethe JS, Heringa J, 't Hoen PA, Hooft R, Kuhn T, Kok R, Kok J, Lusher SJ, Martone ME, Mons A, Packer AL, Persson B, Rocca-Serra P, Roos M, van Schaik R, Sansone SA, Schultes E, Sengstag T, Slater T, Strawn G, Swertz MA, Thompson M, van der Lei J, van Mulligen E, Velterop J, Waagmeester A, Wittenburg P, Wolstencroft K, Zhao J, Mons B. 2016. The FAIR Guiding Principles for scientific data management and stewardship. Scientific data 3:160018.

Yu J, Zhang B, Zhang Y, Xu C-Q, Zhuo W, Ge J, Li J, Gao N, Li Y, Yang M. 2018. A bindingblock ion selective mechanism revealed by a $\mathrm{Na} / \mathrm{K}$ selective channel. Protein $\&$ cell 9:629-639. 
Figure 1

Scheme of score calculation 


\section{Protein Data Bank (PDB) data base \\ 1178}

E. coli Protein structures

(ECORI)

Human Protein structures

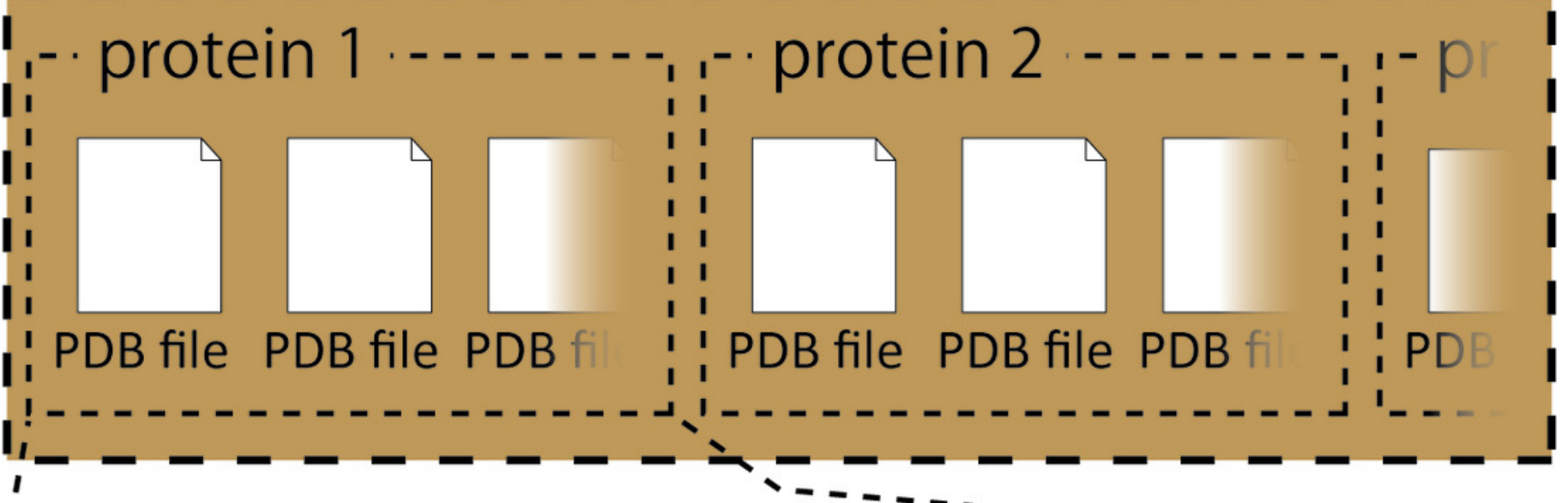

I- - - - - - - - - - - - - - - - - - - - - - -

'- chain 1 from PDB file- $-_{\boldsymbol{1}_{-}}$chain 2 from PDB file- - "- cha !

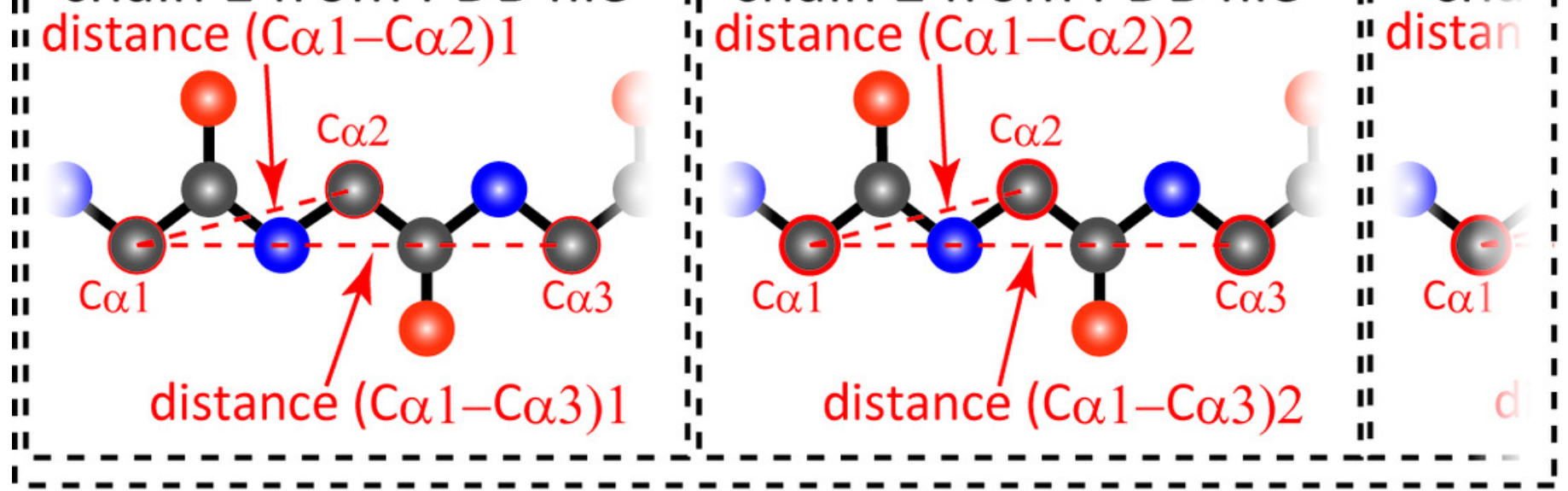

Score $\left(\mathrm{C}_{\alpha 1} 1-\mathrm{C}_{\alpha 2}\right)=\frac{\overline{\text { distance between } \mathrm{C} \alpha 1-\mathrm{C}_{\alpha 2}}}{\text { Stdev. of distance between } \mathrm{C} \alpha 1-\mathrm{C}_{\alpha 2}}$ 


\section{Figure 2}

The summary plot of xUMFs

(A) log-log plot of ECOLI set against analyzed chain length. Five COP9 proteins are colored red. (B) semi-log plot of ECOLI proteins against average resolution. Ten out of 52 ribosomal proteins are colored red, showing the UMF range roughly from 25 to 70. (C) log-log plot of human proteins against analyzed chain length. (D) semi-log plot of human proteins against average resolution $(\AA)$. Five COP9 proteins are colored red.

A

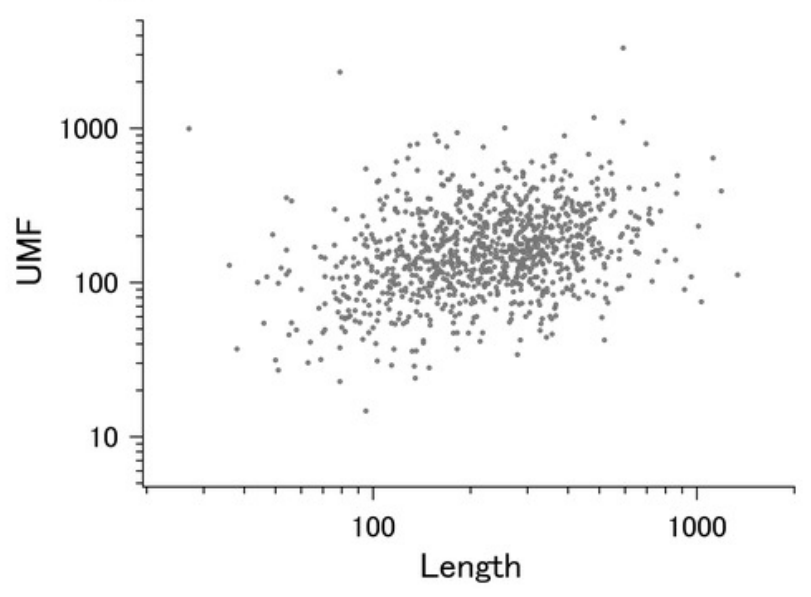

$\mathrm{C}$

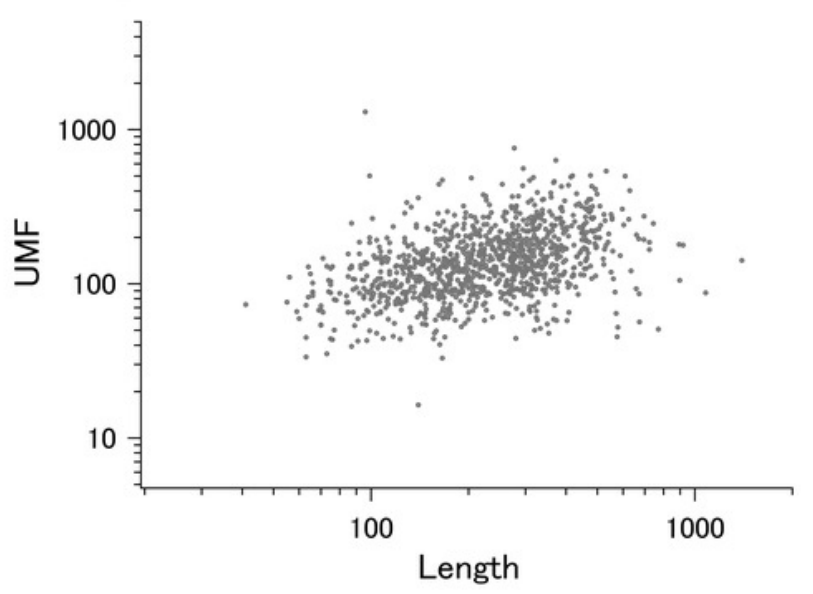

B

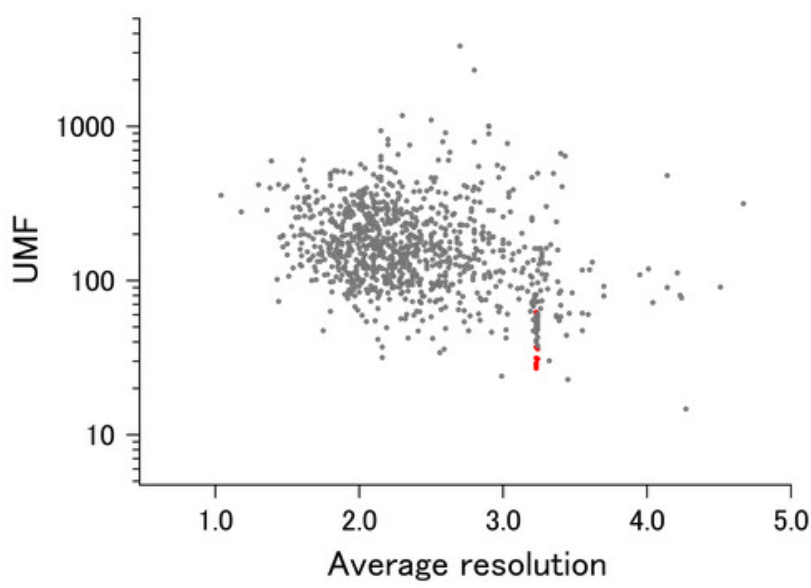

D

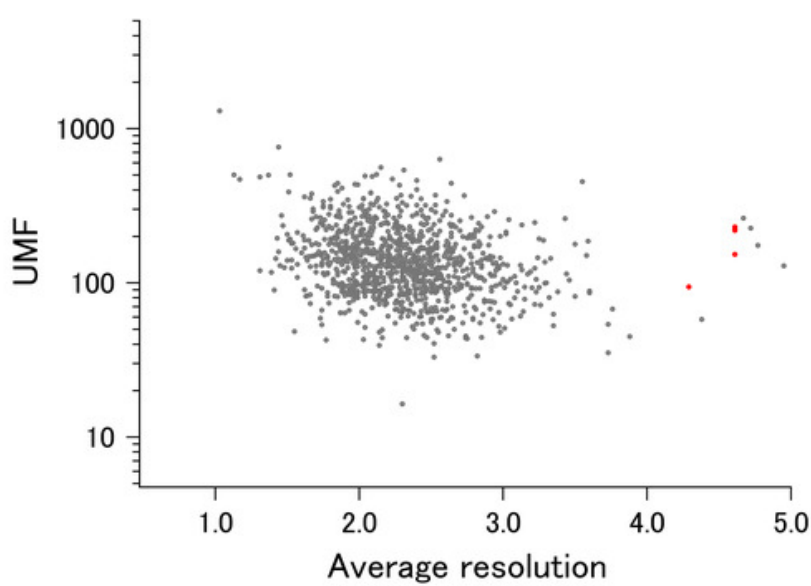


Figure 3

The distribution plot of xUMFs.

Red: ECOLI set. Gray: human proteins.

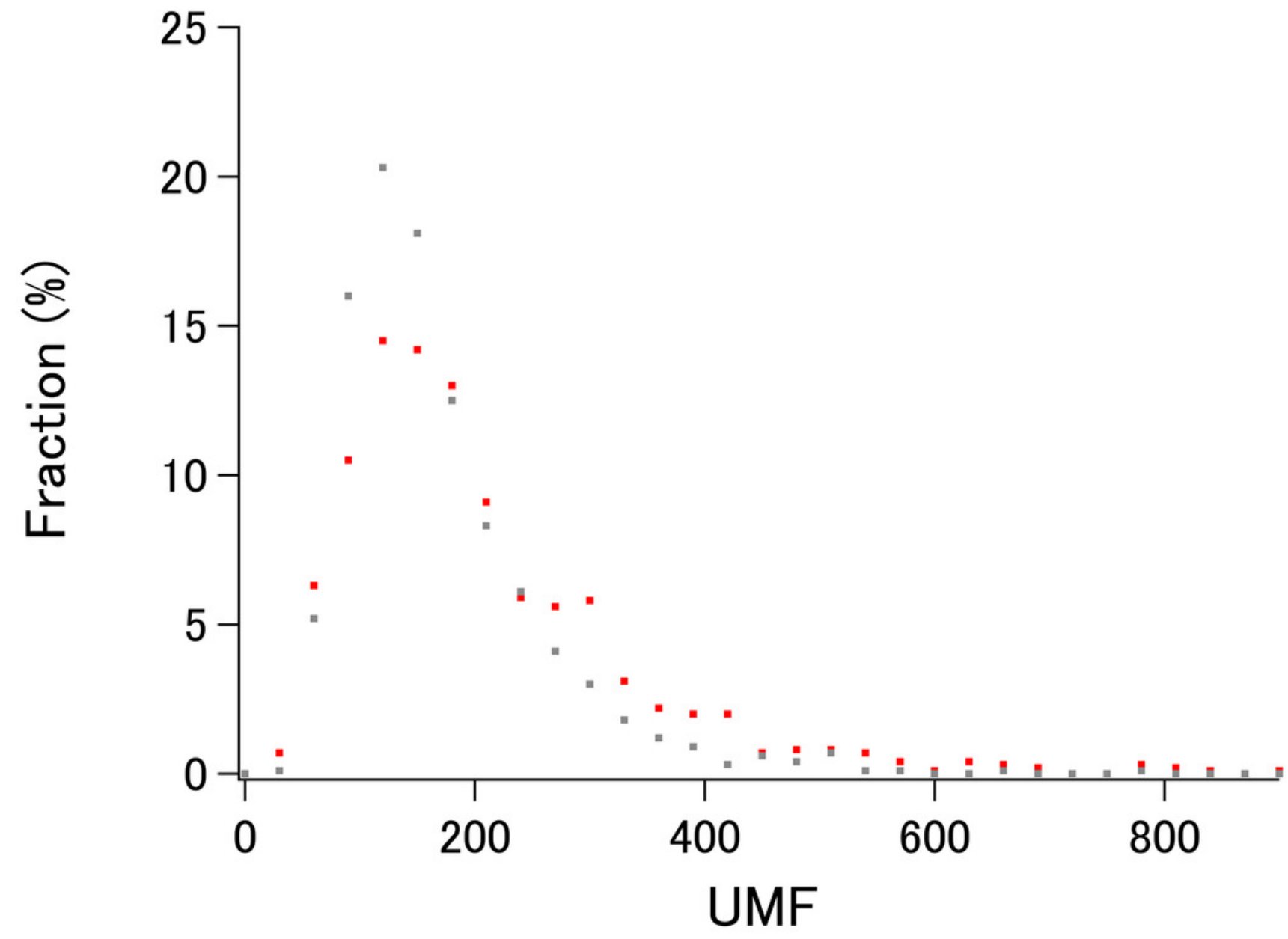


Figure 4

The summary plot of xUMFs and eUMFs.

(A) log-log plot of ECOLI set against analyzed chain length. (B) log-log plot of ECOLI set against average resolution. eUMFs are shown as red squares. 

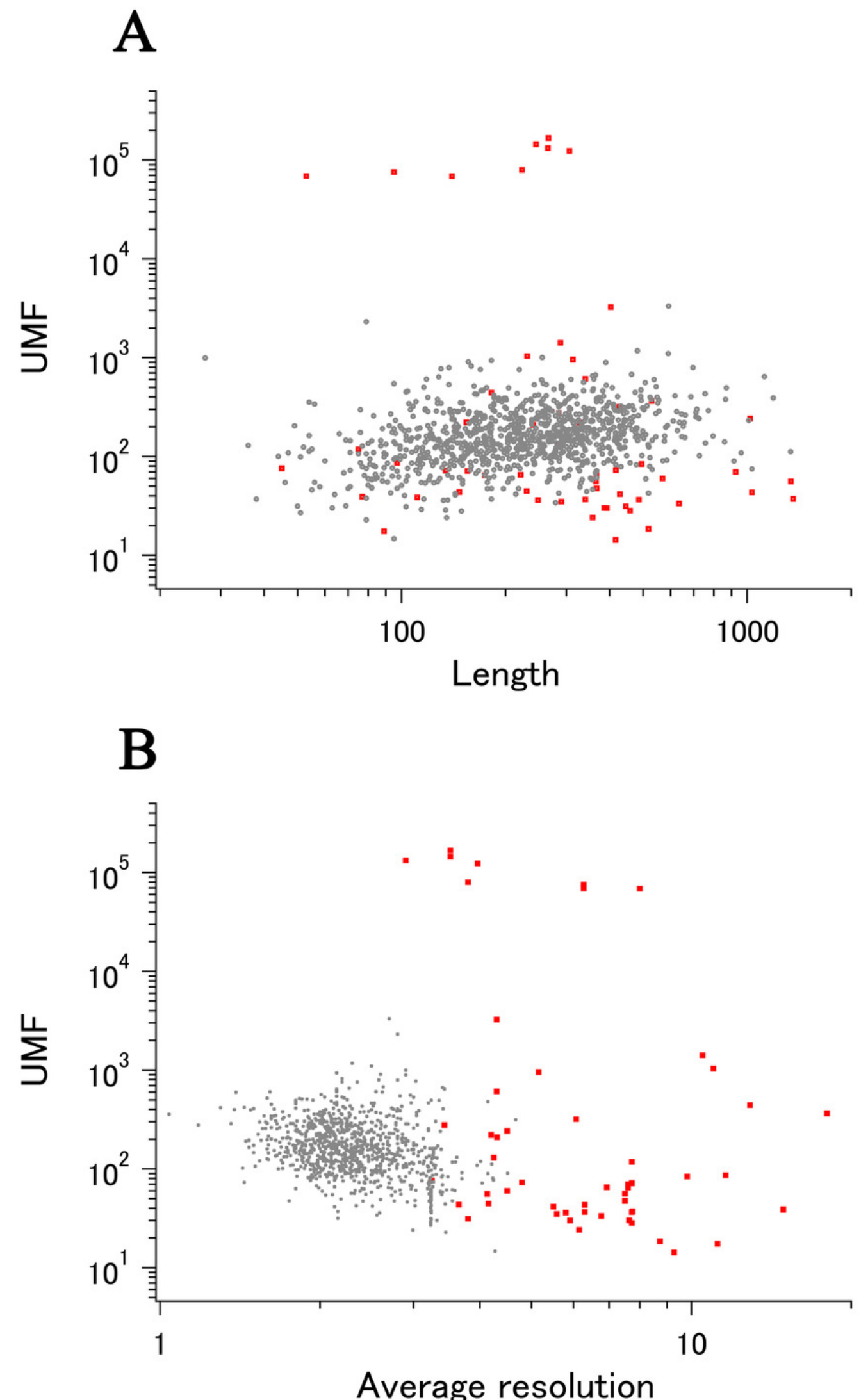


\section{Figure 5}

The semi-log main plot obtained from DSA.

(A) Ynal of ECOLI (cryo-EM). (B) MscS of ECOLI by single entry analysis (X-ray). (C) MscS of ECOLI by multi entry analysis (X-ray). (D) CNR1 of human (X-ray). The unit of average distance is $\AA$.
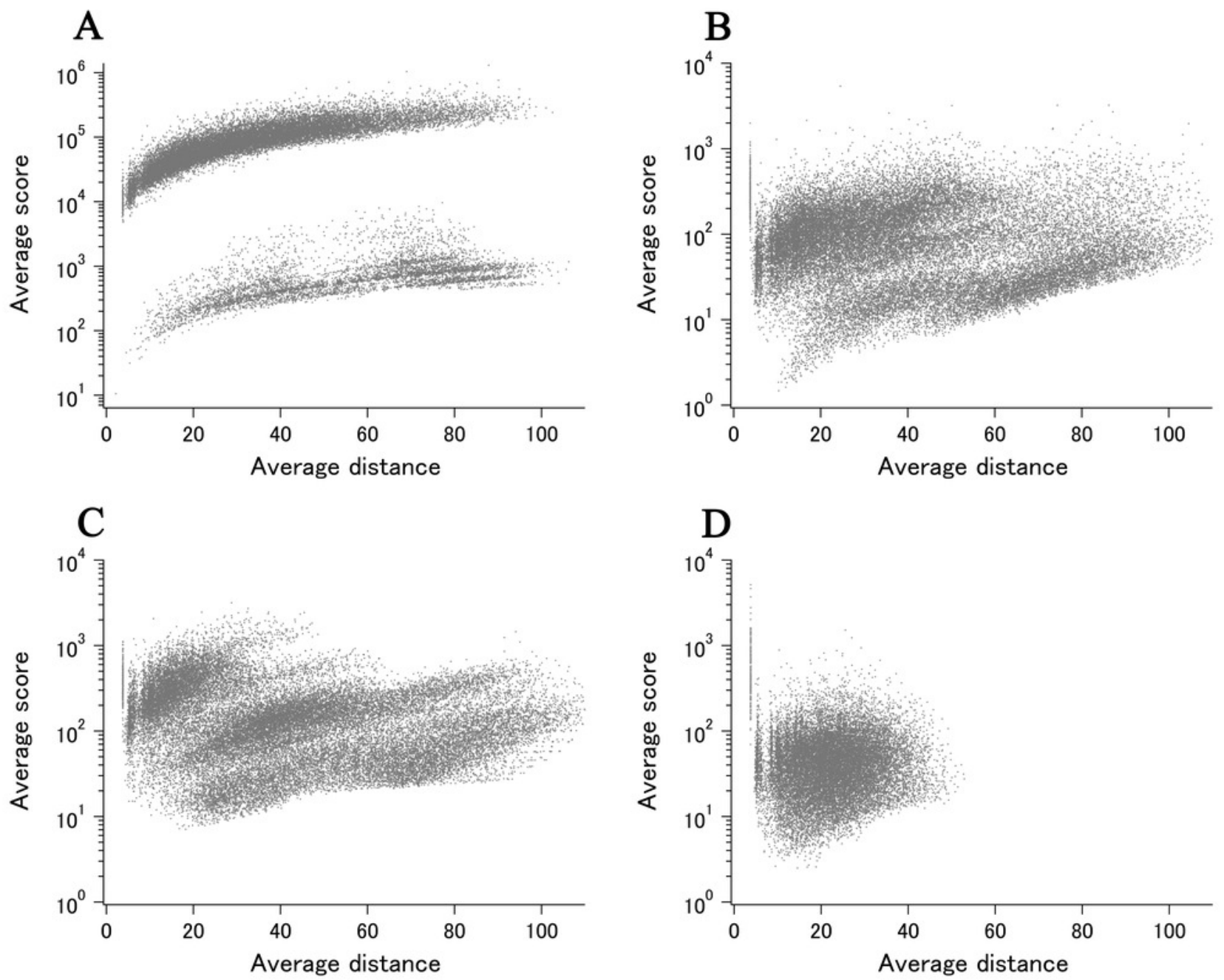
Figure 6

The main plot obtained from DSA.

(A) Ynal of ECOLI (cryo-EM). (B) prepilin peptidase-dependent pilin of ECOLI (cryo-EM). (C) hist1h2ab of human (X-ray). (D) hist1h2ab of human (cryo-EM).
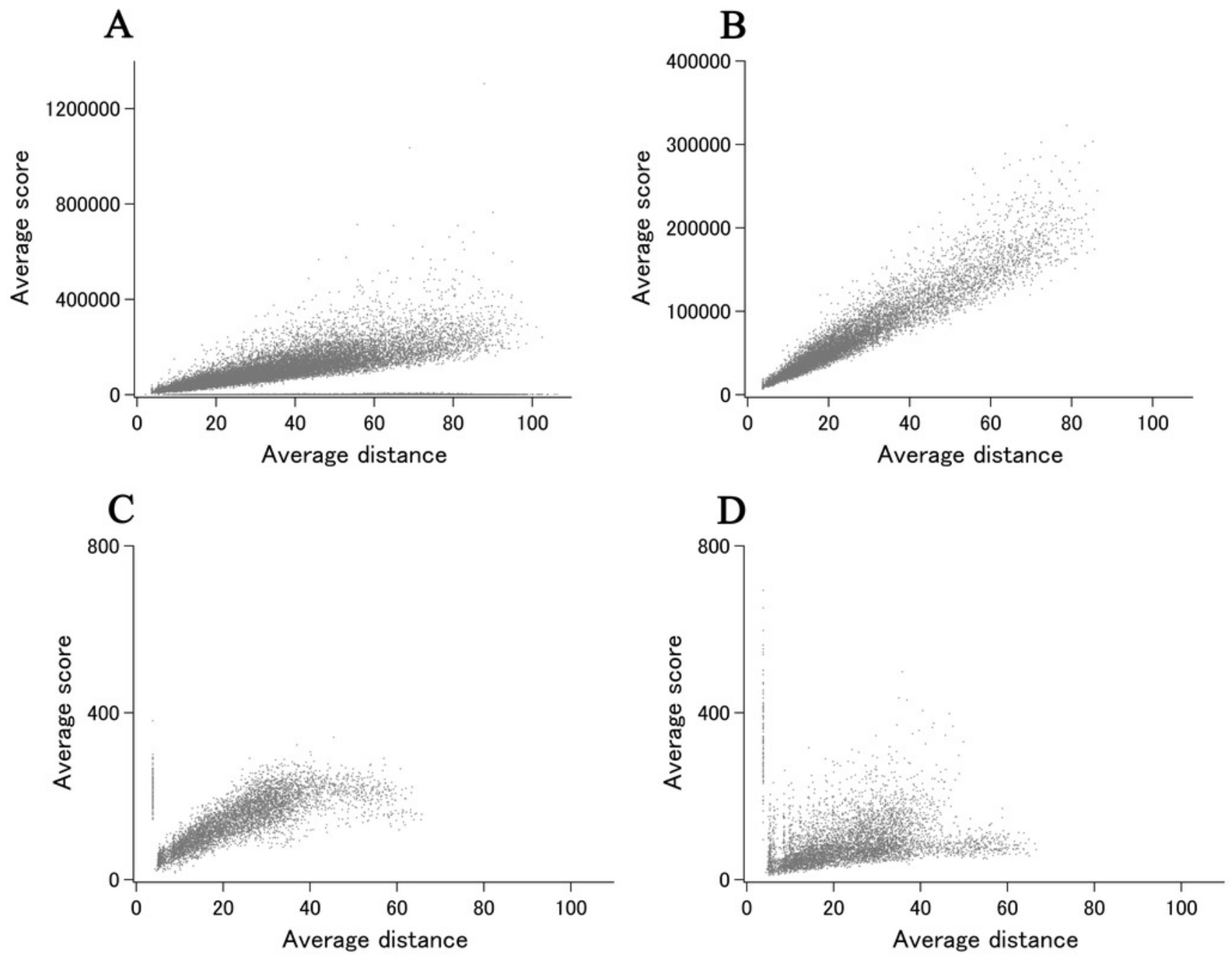
Figure 7

Recent examples of DSA update.

(A) Progress plot of YedK (P76318/A0A2S5ZH06, the data point of only P76318 is shown in red.). (B) semilog main plot of YedK (P76318). (C) Progress plot of YedK (P76318/A0A2S5ZH06, the third data point in A is moved to the $7^{\text {th }}$ position). (D) semi-log main plot of YedK (P76318/A0A2S5ZH06).
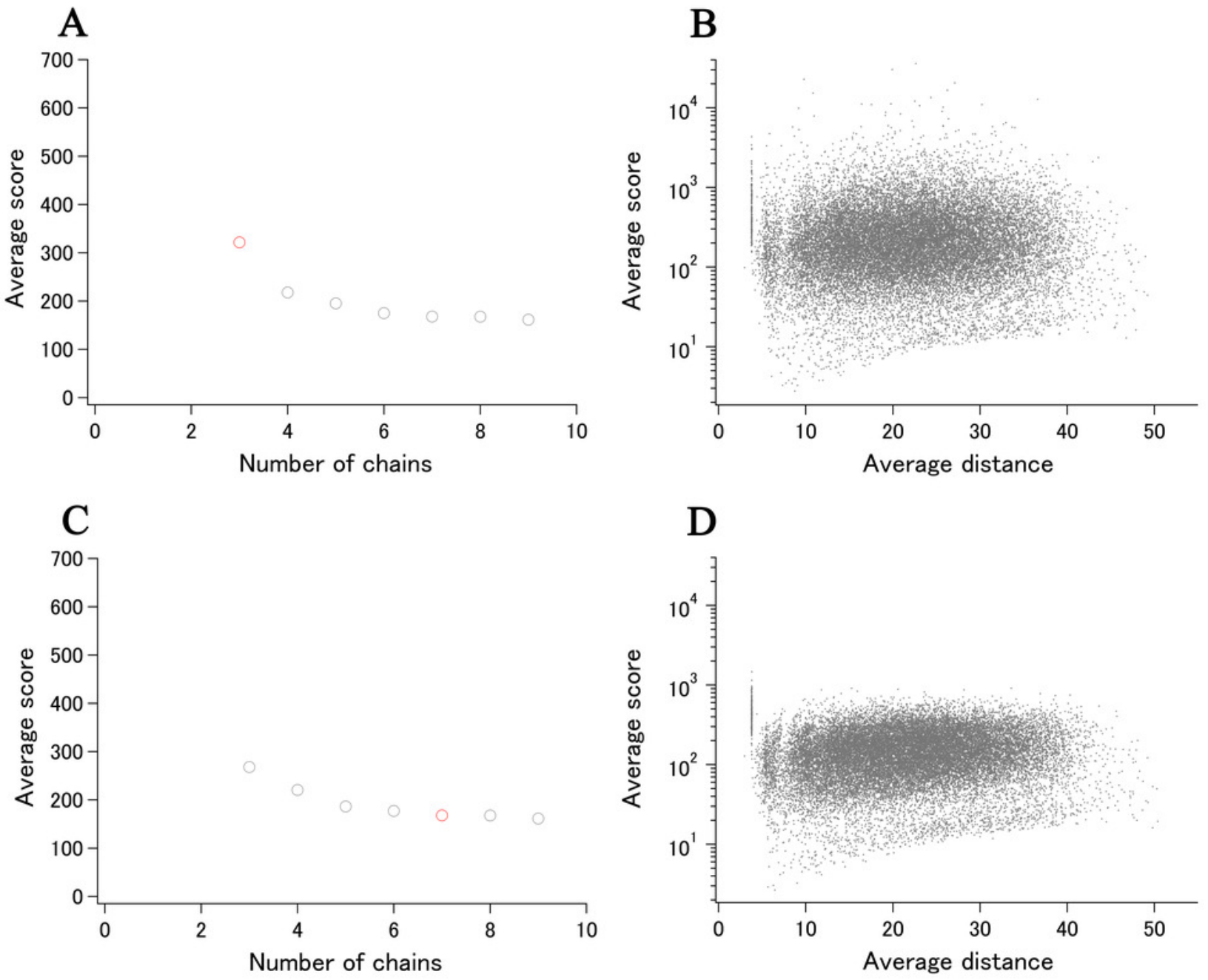